\title{
Linear IC detectors for low to medium SNR ill-conditioned communication systems with unknown noise variance
}

\author{
Abdelouahab Bentrcia ${ }^{*}$ and Saleh A Alshebeili ${ }^{1,2}$
}

\begin{abstract}
In this paper, we introduce two new linear parallel interference cancellation (LPIC) detectors that are suitable for low to medium signal-to-noise ratio ill-conditioned communication systems and do not require knowledge of the noise variance but perform close to the linear minimum mean square error detector, which needs such information. Particularly, we focus in this work on fast linear parallel interference cancellation detectors that are asymptotically equivalent to the steepest descent and conjugate gradient algorithms, respectively, and show that they exhibit a spectral filtering property and semi-convergence behavior. Consequently, a deterministic stopping rule to stop the LPIC iterations that is independent of the noise level (known as the L-curve method) is investigated and tested. Simulation results are presented to support our theoretical findings.
\end{abstract}

Keywords: Linear; PIC; Regularization; Semi-convergence; Linear MMSE; L-curve

\section{Introduction}

The capacity of the third-generation cellular systems and optical networks using optical CDMA (OCDMA) technology is mainly limited by the multi-access interference (MAI) [1]. Other systems suffer from other types of interference such as the inter-carrier interference (ICI) in orthogonal frequency division multiple access (OFDMA) and inter-antenna interference (IAI) in multiinput multioutput (MIMO) systems, just to name a few [1].

In $4 \mathrm{G}$ and beyond wireless communication systems, the problem of interference is becoming of increasing importance because cells are getting smaller and condensed (i.e., femto-cells) and new technologies that cause additional interference are introduced. For example, relay nodes are proposed to increase coverage and allow cooperative communication; however, they also bring additional interference into the network [2].

To combat these different types of interferences, various interference cancellation and multiuser detection algorithms are proposed. Multiuser detectors (MUDs) are mainly introduced to reduce the effect of interference in

\footnotetext{
* Correspondence: abentrcia@ksu.edu.sa

'Prince Sultan Advanced Technologies Research Institute (PSATRI), King Saud University, P.O. Box 800, Riyadh 11421, Saudi Arabia

Full list of author information is available at the end of the article
}

wireless/wired systems and consequently to boost the system capacity and throughput. Many multiuser detectors were developed in the literature and have found applications in various wireless/wired systems such as OCDMA, MIMO-OFDM, and MIMO-UWB, just to name a few [1-5]; however, due to the fact that the capacity of CDMA systems is essentially limited by MAI, a large part of the literature of MUDs focused on systems based on the CDMA technology.

The decorrelating detector is an effective multiuser detector to eliminate interference. It is also an important building block for nonlinear multiuser detectors. It enjoys several desirable features such as: (1) complete removal of interference and (2) independence of noise level information. The latter is very important in situations where the estimation of the noise variance is not possible or not accurate. This is the case where the noise term includes in addition to thermal noise, other types of background noise such as co-channel interference which may change significantly with time/frequency (particularly in frequencyhopping systems) and its variance can be considered as unknown to the receiver [6,7].

However, the decorrelating detector suffers from two drawbacks: (1) Its relatively high computational complexity which is of the order of $O\left(N^{3}\right)[1]$, where $N$ in the 
dimension of the system's cross-correlation matrix and (2) noise enhancement effect. Therefore, the challenge is to maintain the advantages of the decorrelating detector and overcome its deficiencies, that is, to lower its computational complexity and combat the noise enhancement effect but without requiring the knowledge of the noise variance, like the linear minimum mean square error (LMMSE) detector.

Recent results reported in [8] proved the semiconvergence behavior of the conventional linear parallel interference cancellation (LPIC) detector and showed that early stopping rules can be used to combat the noise enhancement effect. They used the Morozov discrepancy rule to stop the LPIC iterations prior to final convergence in order to avoid noise magnification. However, the conventional LPIC detector is very slow and may require a very large number of stages to converge especially for illconditioned communication systems. Moreover, the Morozov discrepancy rule requires the noise level information and therefore cannot be used in many practical settings.

Building up on these results, a twofold approach is proposed in this work to overcome the drawbacks of the decorrelator detector cited above. First, we employ fast linear interference cancellation detectors to reduce its computational complexity, and then, we make use of an early stopping technique (known as the L-curve method) that does not require noise level information to reduce the noise enhancement effect [9]. Up to our knowledge, this is the first work that investigates the possible use of these early stopping rules that do not require noise level information in the communication field. Preliminary results are promising and suggest that more improvements and extensions can be made.

The organization of this paper is as follows: in Section 2, a simplified system model of the OFDMA uplink that will be used throughout this work is briefly described. In Section 3, the decorrelator detector's solution is analyzed and some of the regularization techniques that are used to overcome its drawbacks are detailed. Section 4 briefly describes the fast LPIC detectors and shows their spectral filtering property. Section 5 analyzes the semi-convergence behavior of the LPIC detector and then investigates the L-curve stopping rule and shows how it can be applied to the LPIC detector. Section 6 supports the theoretical findings by a number of simulations. Finally, Section 7 concludes the paper with some results and recommendations.

\section{System model}

For illustration purposes only and similar to [8], we consider an uplink OFDMA system where $K$ users transmit simultaneously over a Rayleigh fading channel using quadrature phase-shift keying (QPSK). Particularly, we consider in this work the effect of ICI due to the misalignment of the carrier frequencies and to the Doppler shifts of different users such as in the uplink of the mobile WIMAX
(IEEE 802.16 Wireless MAN standard) [10]. This is depicted in Figure 1.

In this work, we consider the interleaved subcarrier allocation scheme because it is well known that this scheme suffers the most from ICI compared to other subcarrier allocation schemes [11].

An OFDM symbol consisting of $N_{u}$ samples with sampling time $T_{u}$ where $N_{u}$ is the total number of data samples is transmitted using $N$ orthogonal subcarriers. Without loss of generality, we assume that the total number of subcarriers of the IFFT matrix $\Psi$ with elements $\Psi_{n_{u}, n}=\frac{1}{\sqrt{N_{u}}} e^{\frac{j 2 \pi n_{u} n}{N_{u}}}, 1 \leq n_{u} \leq N_{u}$, and $1 \leq n \leq N$ is divided equally among all users; therefore, the total number of subcarriers per user is $N_{k}=N / K$.

Based on Figure 1, the received signal $\mathbf{r}(m)$ at the $m$ th OFDM symbol is expressed in vector-matrix form as:

$$
\begin{aligned}
\mathbf{r}(m) & =\tilde{\mathbf{\Psi}} \mathbf{H}(m) \mathbf{A b}(m)+\mathbf{n}(m) \\
& =\tilde{\tilde{\mathbf{\Psi}}}(m) \mathbf{b}(m)+\mathbf{n}(m),
\end{aligned}
$$

where

- $\tilde{\boldsymbol{\Psi}}=\Psi_{\circ}\left(\mathbf{1}_{1, N_{k}} \otimes \mathbf{E}\right)$ is a combination of the IFFT matrix $\Psi$ and the normalized carrier frequency offset (NCFO) matrix E. Here, ${ }^{\circ}$ and $\otimes$ denote the Schur and Kronecker products, respectively, and $\mathbf{1}_{1, N_{k}}$ denotes a 1-by- $N_{k}$ vector of ones. The NCFO matrix can be partitioned as $\quad \mathbf{E}=\left[\begin{array}{llll}\boldsymbol{\varepsilon}_{1} & \boldsymbol{\varepsilon}_{2} & \cdots\end{array}\right.$ $\left.\begin{array}{lll}\boldsymbol{\varepsilon}_{k} & \cdots & \boldsymbol{\varepsilon}_{K}\end{array}\right]$, where the vector $\boldsymbol{\varepsilon}_{k}$ is given

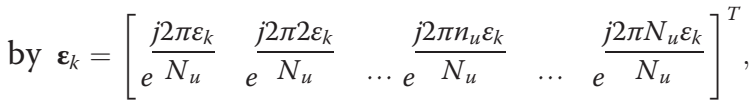
where $\varepsilon_{k}=\Delta f_{k} / \Delta f$ is the NCFO of the $k$ th user and $\Delta f$ is the subcarrier spacing.

- $\mathbf{H}(m)$ is the matrix of Rayleigh fading coefficients and it is given by: $\mathbf{H}(m)=\operatorname{diag}\left(\mathbf{H}_{1}(m) \quad \mathbf{H}_{2}(m) \quad \cdots\right.$

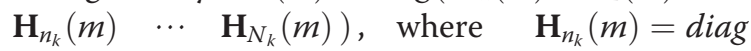
$\left(h_{1, n_{\mathrm{k}}}(m) \mathrm{h}_{2, n_{k}}(m) \cdots h_{k, n_{k}}(m) \quad \cdots \quad h_{K, n_{k}}(m)\right)$.

- A is the matrix of amplitudes and it is given by: $\mathbf{A}=\operatorname{diag}\left(\begin{array}{llllll}\mathbf{A}_{1} & \mathbf{A}_{2} & \cdots & \mathbf{A}_{n_{k}} & \cdots & \mathbf{A}_{N_{k}}\end{array}\right), \quad$ where $\mathbf{A}_{n_{k}}=\operatorname{diag}\left(\begin{array}{llllll}a_{1, n_{k}} & a_{2, n_{k}} & \cdots & a_{k, n_{k}} & \cdots & a_{K, n_{k}}\end{array}\right)$. It is used to weight the signals of different users with different powers to simulate near-far scenarios.

- $\mathbf{b}(m)$ is the vector of transmitted data symbols and it can be partitioned as: $\mathbf{b}(m)=\left[\begin{array}{lll}\mathbf{b}_{1}(m) & \mathbf{b}_{2}(m) & \cdots\end{array}\right.$ $\left.\begin{array}{lll}\mathbf{b}_{n_{k}}(m) & \cdots & \mathbf{b}_{N_{k}}(m)\end{array}\right]^{T}$, where $\mathbf{b}_{k}(m)=\left[\begin{array}{ll}b_{1, n_{k}} & (m)\end{array}\right.$ $\left.b_{2, n_{k}}(m) \cdots b_{k, n_{k}}(m) \quad \cdots \quad b_{K, n_{k}}(m)\right]$.

- $\mathbf{n}(m)$ is an $N$-length vector of independently and identically distributed additive white Gaussian noise samples with zero mean and variance $\rho^{2}$.

And finally, the combination $\tilde{\tilde{\mathbf{\Psi}}}(m)=\tilde{\boldsymbol{\Psi}} \mathbf{H}(m) \mathbf{A}$ is the system matrix and it results from the multiplication of 


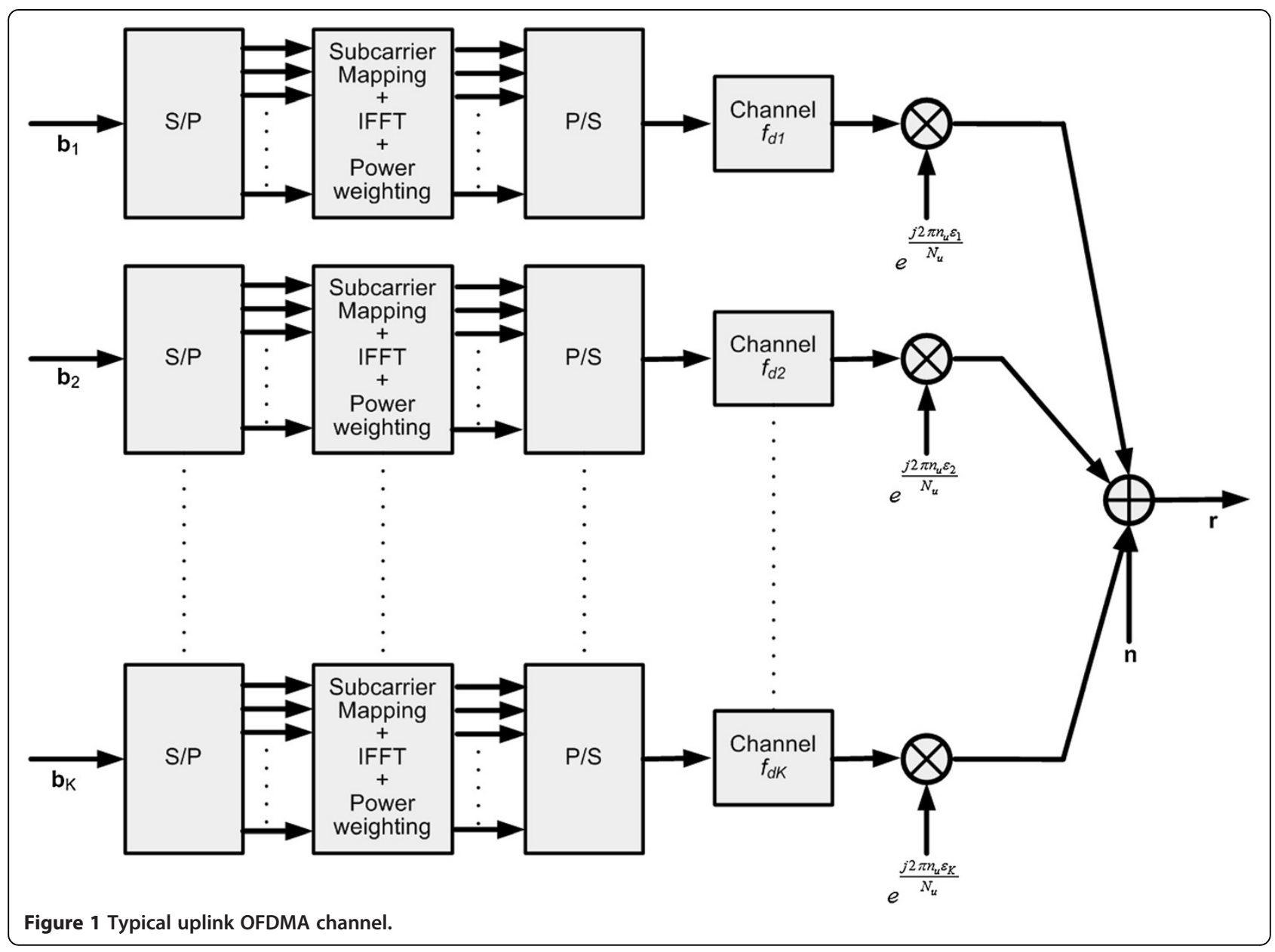

the IFFT, the NCFO, the channel gain, and power weighting matrices, respectively. To simplify the notations, we drop in all subsequent equations the OFDM symbol index $m$ from all matrices and vectors used in Equation (1).

\section{Regularization techniques for combating the noise enhancement effect}

The decorrelator detector (known also as the zeroforcing detector) is a fundamental detector that allows complete removal of the interference, and it results from solving the following least square minimization problem:

$$
\mathbf{b}^{*}: \min _{\mathbf{b} \in \mathbb{C}^{N}}\|\mathbf{r}-\tilde{\tilde{\Psi}} \mathbf{b}\|_{2}^{2}
$$

which yields

$$
\mathbf{y}^{\mathrm{DEC}}=\tilde{\tilde{\boldsymbol{\Psi}}}^{\dagger} \mathbf{r}=\left(\tilde{\tilde{\boldsymbol{\Psi}}}^{H} \tilde{\tilde{\boldsymbol{\Psi}}}\right)^{-1} \tilde{\tilde{\Psi}}^{H} \mathbf{r}
$$

where $\tilde{\tilde{\Psi}}^{\dagger}$ is the pseudo-inverse of the system matrix $\tilde{\tilde{\Psi}}$ and $\tilde{\tilde{\Psi}}^{H} \tilde{\tilde{\Psi}}$ is the ICI matrix.
Using the singular value decomposition (SVD), the system matrix $\tilde{\tilde{\Psi}}$ can be decomposed as:

$$
\tilde{\tilde{\mathbf{\Psi}}}=\mathbf{U} \boldsymbol{\Sigma} \mathbf{V}^{H}
$$

where $\mathbf{U}$ and $\mathbf{V}$ are the $N$-by- $N$ and $N$-by- $N$ unitary matrices that represent the left and right singular vectors of $\tilde{\tilde{\Psi}}$, respectively, and $\Sigma$ is an $N$-by- $N$ diagonal matrix with elements $\sigma_{k}, 1 \leq n \leq N$ that represent the singular values of $\tilde{\tilde{\Psi}}$. In addition, the unitary matrices $\mathbf{U}$ and $\mathbf{V}$ can be partitioned as $\mathbf{U}=\left[\begin{array}{llllll}\mathbf{u}_{1} & \mathbf{u}_{2} & \cdots & \mathbf{u}_{n} & \cdots & \mathbf{u}_{N}\end{array}\right]$ and $\mathbf{U}=\left[\begin{array}{ll}\mathbf{v}_{1} & \mathbf{v}_{2} \cdots\end{array}\right.$ $\left.\begin{array}{lll}\mathbf{v}_{n} & \cdots & \mathbf{v}_{N}\end{array}\right]$, respectively. Therefore, Equation (3) can be written in terms of the SVD of $\tilde{\tilde{\Psi}}$ as:

$$
\mathbf{y}^{\mathrm{DEC}}=\tilde{\tilde{\mathbf{\Psi}}}^{\dagger} \mathbf{r}=\mathbf{V} \boldsymbol{\Sigma}^{-1} \mathbf{U}^{H} \mathbf{r}=\sum_{n}^{N} \frac{\mathbf{u}_{n}^{H} \mathbf{r}}{\sigma_{n}} \mathbf{v}_{n} .
$$

Consequently, its norm can be written as:

$$
\left\|\mathbf{y}^{\mathrm{DEC}}\right\|_{2}=\sqrt{\sum_{n}^{N}\left(\frac{\mathbf{u}_{n}^{H} \mathbf{r}}{\sigma_{n}}\right)^{2}} .
$$


It is clear from Equation (6) that as long as $\left|\mathbf{u}_{n}^{H} \mathbf{r}\right|<\sigma_{n}$ for large $n$, the norm of $\mathbf{y}^{\mathrm{DEC}}$ will not go unbounded. This is what is known as the Picard condition [12]; however, due to the contamination of the received signal with noise, the Fourier coefficients $\left|\mathbf{u}_{n}^{H} \mathbf{r}\right|$ do not decay monotonically to zero, instead they settle at certain level $\rho$ while the singular values decay to zero. Therefore, all singular values below $\rho$ contribute to the noise enhancement effect.

To get rid of the small singular values that may cause the violation of the discrete Picard condition, the decorrelator detector's solution is modified by introducing a filtering matrix $\mathbf{F}$ such that:

$$
\mathbf{y}^{\mathrm{REG}}=\sum_{n}^{N} f_{n} \frac{\mathbf{u}_{n}^{H} \mathbf{r}}{\sigma_{n}} \mathbf{v}_{n}=\mathbf{V F} \Sigma^{-1} \mathbf{U}^{H} \mathbf{r}
$$

where $\mathbf{y}^{\mathrm{REG}}$ is the regularized solution and $\mathbf{F}$ is an $N$-by$N$ diagonal matrix with elements $f_{n}$, satisfying:

$$
f_{n} \simeq\left\{\begin{array}{lll}
1 & \text { if } & \sigma_{n} \text { is large } \\
0 & \text { if } & \sigma_{n} \text { is small }
\end{array} \quad, \quad 1 \leq n \leq N\right.
$$

Consequently, these factors filter out solution components pertaining to small singular values.

The most straightforward regularization technique is known as the truncated SVD (TSVD) and is given by [13]:

$$
\mathbf{y}^{\mathrm{TSVD}}=\sum_{n}^{n^{\prime}} \frac{\mathbf{u}_{n}^{H} \mathbf{r}}{\sigma_{n}} \mathbf{v}_{n}
$$

Therefore, the filter factors for this regularization scheme can be expressed as:

$$
f_{n} \simeq\left\{\begin{array}{lll}
1 & \text { if } & n \leq n^{\prime} \\
0 & \text { if } \quad n>n^{\prime}
\end{array} \quad, \quad 1 \leq n \leq N .\right.
$$

This regularization technique is known also as spectral cutting technique and relies on cutting off all solution components under a certain threshold determined by the discrete regularization parameter $n$ '.

Another regularization scheme is known as the Tikhonov regularization technique, and it relies on modifying the least square minimization problem in Equation (2) by penalizing solutions of large norm, that is, [13]:

$$
\mathbf{b}^{*}: \min _{\mathbf{b} \in \mathrm{C}^{N}}\left\{\|\mathbf{r}-\tilde{\tilde{\Psi}} \mathbf{b}\|_{2}^{2}+\eta^{2}\|\mathbf{b}\|_{2}^{2}\right\}
$$

where $\eta$ is the regularization parameter that determines the amount of penalization the solution norm undergoes. The rationale behind this is that from one side we want to make the residual small as in the conventional least squares, but at the same time, we want to obtain meaningful solutions and exclude those with large norms as they are usually contaminated with noise. Fortunately, Equation (11) has a closed form solution and is given by:

$$
\mathbf{y}^{\mathrm{Tik}}=\left(\tilde{\tilde{\mathbf{\Psi}}}^{H} \tilde{\tilde{\mathbf{\Psi}}}+\eta^{2} \mathbf{I}\right)^{-1} \tilde{\tilde{\mathbf{\Psi}}}^{H} \mathbf{r} .
$$

Note that if the regularization parameter is equal to the noise level $\rho$, then the Tikhonov solution equals to the linear minimum mean square error (LMMSE) solution. The filter factors of the Tikhonov regularization scheme are given by:

$$
f_{n}=\frac{\sigma_{n}^{2}}{\sigma_{n}^{2}+\eta^{2}} \simeq\left\{\begin{array}{lll}
1 & \text { if } & \sigma_{n} \gg \eta \\
0 & \text { if } & \sigma_{n} \ll \eta
\end{array} \quad, \quad 1 \leq n \leq N .\right.
$$

The filter factors of the Tikhonov regularization scheme behaves like a smooth low pass filter of the solution terms damping solution components pertaining to singular values less than $\eta$. The filter factors of the TSVD and the Tikhonov regularization schemes are depicted in Figure 2.

Another category of regularization methods is regularization by early stopping [13], that is, apply an iterative method to the least square problem of Equation (2) and stop the iterations prior to convergence. This is motivated by the fact that for ill-conditioned systems, linear iterative methods exhibit a semi-convergence property and tend to generate good solutions at early iterations but after a certain number of iterations, the noise starts dominating the solution and the performance of the iterative method worsens.

Many linear interference cancellation schemes have been shown to be equivalent to certain iterative methods [1], and therefore, they tend to have the same semiconvergence behavior for ill-conditioned systems. Therefore, linear IC detectors equipped with efficient early stopping mechanisms that do not require the noise level information can be used to implement a low-complexity decorrelating detector that resists noise amplification without requiring knowledge of the noise level. In the following, we focus on the LPIC detector and use it as an iterative regularizing scheme to achieve two simultaneous objectives: low-complexity and resistance to noise enhancement.

\section{Intrinsic regularization property of the LPIC detector}

The LPIC detector is an effective scheme for the lowcomplexity approximation of the decorrelator/LMMSE detector [8]. The conventional LPIC detector is asymptotically equivalent to the Jacobi iterative method [1], and therefore, it inherits its slow convergence behavior. Faster LPIC detectors based on faster iterative methods 


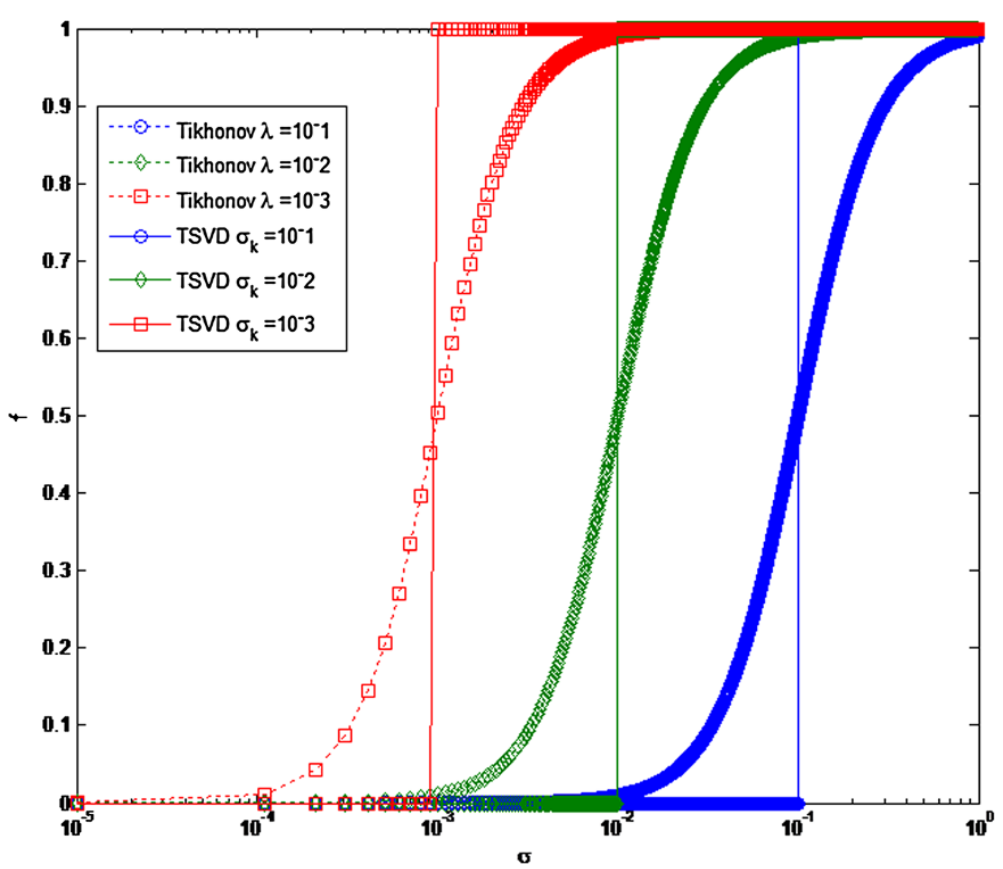

Figure 2 Filter factors of the Tikhonov and TSVD regularization methods.

such as the steepest descent and the conjugate gradient methods are now introduced. The residual norm steepest descent (RNSD) and conjugate gradient least squares (CGLS) methods belong to a class of iterative techniques known as non-stationary iterative methods where the weighting factor changes from one stage to another [14], that is:

$$
\mathbf{y}_{p+1}=\mathbf{y}_{p}+\omega_{p} \mathbf{d}_{p}
$$

where $\omega_{p}$ is the step size and $\mathbf{d}_{p}$ is the search direction.

For RNSD, the search direction is equal to the residual error and is given by: $\mathbf{d}_{p}=\mathbf{e}_{p}=\tilde{\tilde{\Psi}}^{H}\left(\mathbf{r}-\tilde{\tilde{\Psi}}_{\mathbf{y}_{p}}\right)$ while the weighting factor is determined as:

$$
\omega_{p}=\frac{\mathbf{d}_{p}^{H} \mathbf{d}_{p}}{\mathbf{d}_{p}^{H} \tilde{\tilde{\mathbf{\Psi}}}^{H} \tilde{\tilde{\mathbf{\Psi}}} \mathbf{d}_{p}} .
$$

For the CGLS method, the initial search direction $\mathbf{d}_{0}$ is set to the residual error, that is, $\mathbf{d}_{0}=\mathbf{e}_{0}=\tilde{\tilde{\Psi}}^{H}\left(\mathbf{r}-\tilde{\tilde{\Psi}} \mathbf{y}_{0}\right)$, and then, we proceed as:

$$
\omega_{p}=\frac{\mathbf{e}_{p}^{H} \tilde{\tilde{\mathbf{\Psi}}}^{H} \tilde{\tilde{\mathbf{\Psi}}} \mathbf{e}_{p}}{\mathbf{d}_{p}^{H} \tilde{\tilde{\boldsymbol{\Psi}}}^{H} \tilde{\tilde{\mathbf{\Psi}}} \mathbf{d}_{p}}
$$

and

$$
\mathbf{y}_{p+1}=\mathbf{y}_{p}+\omega_{p} \mathbf{d}_{p}
$$

Then, we update $\mathbf{e}_{p}$ and $\mathbf{d}_{p}$ as:

$$
\mathbf{e}_{p+1}=\mathbf{e}_{p}-\omega_{p} \tilde{\tilde{\Psi}} \mathbf{d}_{p}
$$

and

$$
\mathbf{d}_{p+1}=\tilde{\tilde{\mathbf{\Psi}}}^{H} \mathbf{e}_{p+1}+\beta_{p+1} \mathbf{d}_{p}
$$

where

$$
\beta_{p+1}=\frac{\mathbf{e}_{p+1}^{H} \tilde{\tilde{\mathbf{\Psi}}}^{H} \tilde{\tilde{\mathbf{\Psi}}} \mathbf{e}_{p+1}}{\mathbf{e}_{p}^{H} \tilde{\tilde{\mathbf{\Psi}}}^{H} \tilde{\tilde{\mathbf{\Psi}}} \mathbf{e}_{p}} .
$$

To determine the filter factors of the RNSD, it can be shown without loss of generality that if $\mathbf{y}_{0}=0$, then $\mathbf{y}_{p}$ can be written in the following form [14]:

$$
\mathbf{y}_{p+1}=T_{p}^{\mathrm{SD}}\left(\tilde{\tilde{\boldsymbol{\Psi}}}^{H} \tilde{\tilde{\boldsymbol{\Psi}}}\right) \tilde{\tilde{\boldsymbol{\Psi}}}^{H} \mathbf{r}
$$

where the polynomial $T_{p}^{S D}$ is defined as:

$$
T_{p}^{\mathrm{SD}}(\theta)=T_{p-1}^{\mathrm{SD}}(\theta)+\omega_{p}\left(1-\theta T_{p-1}^{\mathrm{SD}}(\theta)\right)
$$

and $T_{-1}^{\mathrm{SD}}(\theta)=0$.

Substituting $\tilde{\tilde{\mathbf{\Psi}}}=\mathbf{U} \boldsymbol{\Sigma} \mathbf{V}^{H}$ into Equation (21), we obtain

$$
\mathbf{y}_{p+1}=\mathbf{V} \mathbf{F} \boldsymbol{\Sigma}^{-1} \mathbf{U}^{H} \mathbf{r}=\sum_{n=1}^{N} \sigma_{n}^{2} T_{p}^{S D}\left(\sigma_{n}^{2}\right) \frac{\mathbf{u}_{n}^{H} \mathbf{r}}{\sigma_{n}} \mathbf{v}_{n},
$$


where $\mathbf{F}$ is $N$-by- $N$ diagonal matrix with elements $f_{p+1, n}$ given by:

$$
f_{p+1, n}=\sigma_{n}^{2} T_{p}^{\mathrm{SD}}\left(\sigma_{n}^{2}\right), \quad 1 \leq n \leq N .
$$

Following the same approach used for the RNSD, the filter factors of the CGLS can be found as:

$$
f_{p+1, n}=\sigma_{n}^{2} T_{p}^{\mathrm{CG}}\left(\sigma_{n}^{2}\right), \quad 1 \leq n \leq N,
$$

where the polynomial $T_{p}^{C G}$ for the CGLS is defined as:

$$
\begin{aligned}
T_{p}^{\mathrm{CG}}(\theta)= & \left(1-\omega_{p} \theta+\frac{\omega_{p} \beta_{p-1}}{\omega_{p-1}}\right) T_{p-1}^{\mathrm{CG}}(\theta)-\frac{\omega_{p} \beta_{p-1}}{\omega_{p-1}} T_{p-2}^{\mathrm{CG}}(\theta) \\
& +\omega_{p}
\end{aligned}
$$

and $T_{-1}^{C G}(\theta)=0$ and $T_{0}^{C G}(\theta)=\omega_{0}$

Finally, the average BER of the LPIC detector based on the RNSD/CGLS iterative methods is given by (see Appendix for derivation):

$$
P_{b}(p)=\frac{1}{N} \sum_{n=1}^{N} \frac{1}{2^{N-1}} \sum_{\substack{\text { alllb } \\ b_{n}=1}} Q\left(\frac{\mathbf{g}_{p, n}^{H} \tilde{\tilde{\Psi}} \mathbf{b}}{\rho \sqrt{\mathbf{g}_{p, n}^{H} \mathbf{g}_{p, n}}}\right),
$$

where $\mathbf{G}_{p}^{H}=T_{p}^{S D}\left(\tilde{\tilde{\mathbf{\Psi}}}^{H} \tilde{\tilde{\mathbf{\Psi}}}\right) \tilde{\tilde{\mathbf{\Psi}}}^{H}$ for the LPIC detector based on the RNSD and $\mathbf{G}_{p}^{H}=T_{p}^{C G}\left(\tilde{\tilde{\mathbf{\Psi}}}^{H} \tilde{\tilde{\mathbf{\Psi}}}\right) \tilde{\tilde{\mathbf{\Psi}}}^{H}$ for the LPIC detector based on the CGLS and $\mathbf{G}_{p}=$ $\left[\mathbf{g}_{p, 1} \mathbf{g}_{p, 2} \cdots \cdots \mathbf{g}_{p, n} \cdots \mathbf{g}_{p, N}\right]$.

\section{Semi-convergence behavior of the LPIC detector and early stopping using the L-curve method}

The LPIC detector exhibits a semi-convergence property where it reaches a BER that is better than that achieved at final convergence. This phenomenon has been studied in more detail in [8]. Interested readers are referred to [8] for more details. For our case, the semi-convergence property of the proposed detectors is illustrated in Figure 3. For the simulation parameters, we set the carrier frequency to $3.5 \mathrm{GHz}$, signalto-noise ratio (SNR) to $13 \mathrm{~dB}, N$ to 128 , and $K$ to 4 with frequency offsets $\left(\varepsilon_{1}=-0.35, \varepsilon_{2}=0.38, \varepsilon_{3}=0.36\right.$, $\left.\varepsilon_{4}=-0.39\right)$ and speeds $80,120,90$, and $100 \mathrm{~km} / \mathrm{h}$, respectively.

It is clear that the two detectors, that is, the one equivalent to the RNSD method (RNSD-LPIC) and the one equivalent to the CGLS method (CGLS-LPIC), exhibit a semi-convergence behavior where they reach their minimum average BER at 10 and 45 stages, respectively, and converge to the decorrelator detector's solution.

The RNSD-LPIC detector exhibits a slower convergence behavior and wide flat minimum while the CGLSLPIC detector exhibits faster convergence and a narrow minimum.

It is difficult to analyze the semi-convergence behavior using the average BER; nevertheless, we can calculate the mean square error between the decision variable of the LPIC detector at stage $(p+1), \mathbf{y}_{p+1}$ and the vector of the transmitted data symbols $\mathbf{b}$, that is:

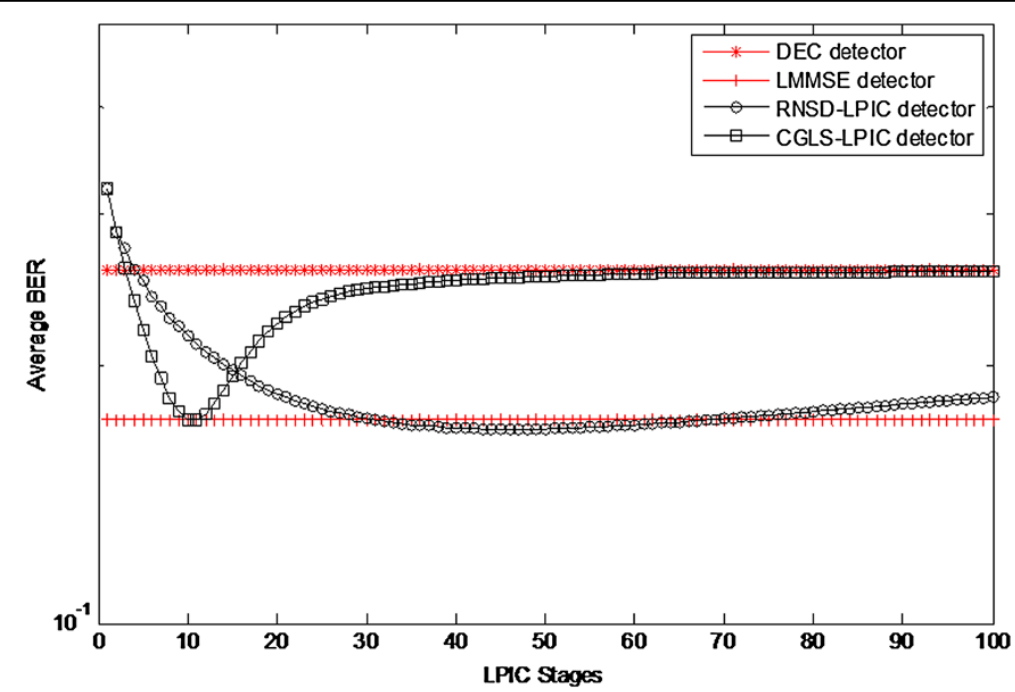

Figure 3 Semi-convergence behavior of the LPIC detector. 


$$
\begin{aligned}
\operatorname{MSE}\left(\mathbf{y}_{p+1}\right) & =\mathrm{E}\left\|\mathbf{y}_{p+1}-\mathbf{b}\right\|_{2}^{2} \\
& =\mathrm{E}\left\|\mathbf{V F} \boldsymbol{\Sigma}^{-1} \mathbf{U}^{H}(\mathbf{b}+\mathbf{n})-\mathbf{V} \boldsymbol{\Sigma}^{-1} \mathbf{U}^{H} \mathbf{b}\right\|_{2}^{2} \\
& =\mathrm{E}\left\|\boldsymbol{\Sigma}^{-1}(\mathbf{F}-\mathbf{I}) \mathbf{U}^{H} \mathbf{b}\right\|_{2}^{2}+\mathrm{E}\left\|\boldsymbol{\Sigma}^{-1} \mathbf{F} \mathbf{U}^{H} \mathbf{n}\right\|_{2}^{2} \\
& =\sum_{n=1}^{N}\left(f_{p+1, n}-1\right)^{2} \frac{\left(\mathbf{u}_{n}^{H} \mathbf{b}\right)^{2}}{\sigma_{n}^{2}}+\rho^{2} \sum_{n=1}^{N} \frac{f_{p+1, n}^{2}}{\sigma_{n}^{2}} \\
& =\underbrace{\left\|\mathbf{b}-\mathrm{E}\left(\mathbf{y}_{p+1}\right)\right\|_{2}^{2}}_{\text {squared bias }}+\underbrace{\mathrm{E}\left\|\mathbf{y}_{p+1}-\mathrm{E}\left(\mathbf{y}_{p+1}\right)\right\|_{2}^{2}}_{\text {variance }}
\end{aligned}
$$

It can be seen that the MSE can be decomposed into two components: the bias (also known as the data error) and the variance (also known as the noise error). The data error is caused by using a modified inverse of the ICI matrix $\tilde{\tilde{\Psi}}^{H} \tilde{\tilde{\Psi}}$ instead of the true inverse whereas the noise error is caused by the noise enhancement effect. It is evident from Equation (28) that if $\mathbf{F} \simeq \mathbf{I}$, the data error is small but the noise error is large due to the noise enhancement effect; however, if $\mathbf{F} \simeq \mathbf{0}$, the noise error is small but the data error is large, and as a result, the solution is heavily damped and a large part of it is lost.

Therefore, a proper choice of the filtering matrix $\mathbf{F}$ should balance between the data and noise errors. Because the amount of filtering introduced by the filtering matrix is proportional to the stage index $p$, a proper stopping rule needs to be devised. Many stopping rules have been developed in the literature but roughly they can be classified into three broad techniques [9]:

- Methods requiring the knowledge of the exact noise level.

- Methods requiring the knowledge of the approximate noise level.

- Methods not requiring the knowledge of the noise level.
Due to the fact that we are assuming the absence of the noise level information, we neglect the first two categories and focus on the last one. Under this category, the most known stopping rule technique is the L-curve method [15]. This method has been used successfully in many areas such as spectroscopy, seismography, and medical imaging. The L-curve method exhibits some desirable features such as:

- Does not need the noise level information. This is important for communication systems where this information is not always available or it is not accurate.

- Works well under colored Gaussian noise. This is important when the additive white Gaussian noise assumption in communication systems is violated, or it becomes colored because of some signal processing operations such as matched filtering.

This method intends to balance between two conflicting goals: minimizing the residual error norm and keeping the solution norm small. Careful inspection reveals that the norm of the residual error $\left\|\mathbf{r}-\tilde{\tilde{\Psi}} \mathbf{y}_{p}\right\|_{2}$ declines sharply at early stages, and then, it flattens while the solution norm $\left\|\mathbf{y}_{p}\right\|_{2}$ increases sharply at early stages and then it flattens. Consequently, if we plot the residual error norm versus the solution norm, we obtain an Lshaped curve with usually a distinct corner that splits the curve into two separate regions as shown in Figure 4.

Noting that the residual and the solution norms can be written in terms of the filtering factors as [13]:

$$
\left\|\mathbf{r}-\tilde{\tilde{\mathbf{\Psi}}} \mathbf{y}_{p}\right\|_{2}^{2}=\sum_{n=1}^{N}\left(\left(1-f_{p, n}\right) \mathbf{u}_{n}^{H} \mathbf{r}\right)^{2}
$$

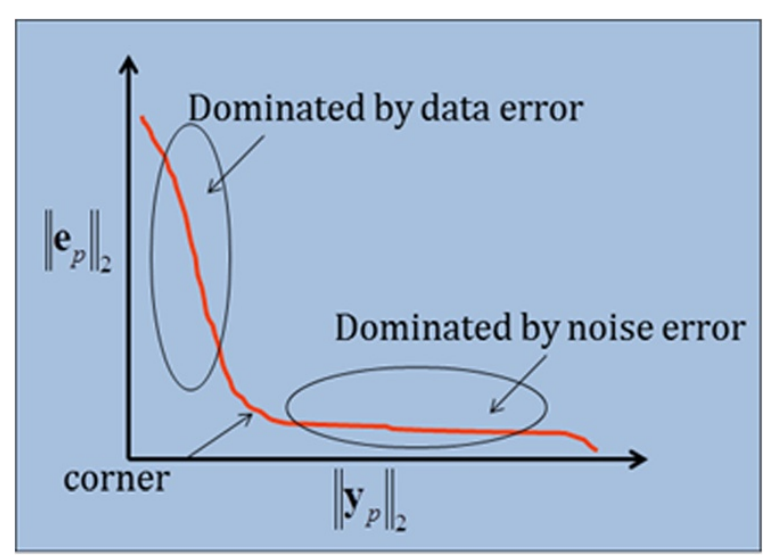

Figure 4 The L-curve for the LPIC detector. 
and

$$
\left\|\mathbf{y}_{p}\right\|_{2}^{2}=\sum_{n=1}^{N}\left(f_{p, n} \frac{\mathbf{u}_{n}^{H} \mathbf{r}}{\sigma_{n}}\right)^{2}
$$

respectively. It is clear that if $f_{p, n} \approx 1$ (at early stages), we are in the upper part of the curve. A small change in $f_{p, n}$ leads to a larger change in $f_{p, n}^{2}$ than in $\left(1-f_{p, n}\right)^{2}$; hence, we expect $\left\|\mathbf{y}_{p}\right\|_{2}$ to change the most in this region, i.e., we expect the curve to be steep. On the other hand, if $f_{p, n} \approx 0$ (at late stages), we are in the lower part of the curve. A small change in $f_{p, n}$ leads to a larger change in $\left(1-f_{p, n}\right)^{2}$ than in $f_{p, n}^{2}$; hence, we expect $\left\|\mathbf{r}-\tilde{\tilde{\Psi}} \mathbf{y}_{p}\right\|_{2}$ to change the most in this region, i.e., we expect the curve to be flat here.

The two regions, that is, the vertical and horizontal, are dominated by two types of errors: data error and noise error, respectively. The corner formed by the conjunction of these two regions balances between the data and noise errors, and therefore, the stage index corresponding to this corner is selected as the best regularization parameter that compromises between data and noise errors.

Many algorithms have been devised to find the corner of the L-curve [16]. The most common technique is known as the maximum curvature method. It consists of choosing the stage index that maximizes the following function:

$$
p_{\mathrm{opt}}=\underset{1 \leq p \leq P}{\arg \max }\left\{\frac{\rho(p)^{\prime \prime} \eta(p)^{\prime}-\rho(p)^{\prime} \eta(p)^{\prime \prime}}{\left(\rho(p)^{\prime 2}+\eta(p)^{\prime 2}\right)^{\frac{3}{2}}}=\kappa(p)\right\},
$$

where $\eta(p)=\left\|\mathbf{y}_{p}\right\|_{2}$ and $\rho(p)=\left\|\mathbf{r}-\tilde{\tilde{\Psi}} \mathbf{y}_{p}\right\|_{2}$ and denotes differentiation with respect to the regularization parameter $p$.

The authors in [17] suggested plotting the residual norm versus the stage index $p$ and noticed that this curve usually exhibits an L-curve shape as well. For this curve, one should find the stage index that maximizes the following simplified function:

$$
p_{\text {opt }}=\underset{1 \leq p \leq P}{\arg \max }\left\{\frac{\rho(p)^{\prime \prime}}{\left(\rho(p)^{\prime 2}+1\right)^{\frac{3}{2}}}=\kappa(p)\right\} .
$$

In order to implement the above stopping rule, we have to evaluate the norm of the residual error for each OFDM symbol a certain number of stages till the Lshaped curve is obtained and calculate the curvature information to ultimately determine the optimal stopping stage. This is too expensive in practice and consequently, another alternative should be sought. Fortunately, simulation results reveal that the optimal stage index $p_{\text {opt }}$ is insensitive to variations in the condition number of system matrix and is almost constant from one OFDM symbol to another, and therefore, it is sufficient to obtain $p_{\text {opt }}$ for a few OFDM symbols (practically in the range of 10 to 100 OFDM symbols) and then average it and use it for the rest of the OFDM symbols. This works well in practice and costs only a marginal addition to the overall LPIC computational complexity.

Another problem with the LPIC detector based on the CGLS is that the norm of the residual error $\left\|\mathbf{r}-\tilde{\tilde{\Psi}} \mathbf{y}_{p}\right\|_{2}$ behaves erratically for very ill-conditioned systems [14] where the norm of the residual error may increase occasionally and causes the maximum curvature detector to mistakenly detect the maximum curvature at the wrong stage index. To overcome this problem, we exploit the fact that the residual error decay exponentially and therefore can be fitted by a decaying exponential. The resulting exponentially fitted curve is used instead in computing the maximum curvature and hence the optimal stage index. This method proved to be efficient in combating the erratic behavior of the residual error norm of the LPIC detector based on the CGLS.

Lastly, the L-curve method has obviously some limitations as discussed in [15]. The main one is that the regularized solution does not converge to the true solution as the noise variance vanishes to zero. Therefore, in our application, we expect that the LPIC detector equipped with the L-curve stopping rule will work well for only a certain range of SNRs. Fortunately, this range covers low and medium SNRs where the noise enhancement effect is most prominent. More insight about this issue is given in the simulation results.

\section{Computational complexity}

The computational complexity of the basic LPIC detector with fixed step size used in [8] and the ones proposed here exhibit a computational complexity in the order of $O\left(N^{2}\right)$, namely the conjugate gradient based LPIC detector needs $4 N^{2}+8 N-1$ cflops $^{\text {a }}$ per iteration and the steepest descent based LPIC detector needs $4 N^{2}+6 N-1$ cflops per iteration, and finally, the conventional LPIC detector requires $2 N^{2}+2 N+1$ cflops per iteration. Even though the conjugate gradient based LPIC detector exhibits a slightly higher computational complexity per iteration compared to the other detectors, it however needs the least number of iterations; this is why it is the mostly used iterative method in practice.

Since early stopping methods, whether they require the noise variance information or not can be applied to any LPIC detector, we evaluate in the following the computational complexity of the L-curve early stopping rule and compare it to that proposed in [8] (known as the Morozov early stopping rule). 
The L-curve method requires the following operations with the following computational complexity:

1. Norm calculation of $\eta(p)=\left\|\mathbf{y}_{p}\right\|_{2}: 2 N$ cflops.

2. Norm calculation of $\rho(p)=\left\|\mathbf{r}-\tilde{\tilde{\mathbf{\Psi}}} \mathbf{y}_{p}\right\|_{2} 2 N$ cflops.

3. Curvature information of $\kappa(p): 2 p-3$ flops.

4. Obtaining the index of the maximum curvature: $p_{\text {opt }}=\arg \max _{1 \leq p \leq P}\left\{\frac{\rho(p)^{\prime \prime}}{\left(\rho(p)^{\prime 2}+1\right)^{\frac{3}{2}}}=\kappa(p)\right\}: p$ flops.

Total complexity for $M$ symbols $(M$ in practice is between 10 to 100$)$ is: $4 M N$ cflops $+3 M(p-1)$ flops. On the other hand, the Morozov stopping rule relies mainly on the estimation of the noise variance. A typical noise variance estimation algorithm used in wireless communication systems and specifically within the context of spectrum sensing [18], solves a yule-walker set of equations using Levinson-Durbin algorithm and needs a complexity of $2 M N^{2}$ cflops [19]. It is clear from the above expressions that the computational complexity of the L-curve early stopping method is less than that of Morozov early stopping method by an order of magnitude. Therefore, in terms of computational complexity, using the L-curve, early stopping method is much cheaper than using the Morozov early stopping method.

\section{Simulation results}

In the following, we evaluate the performance of the LPIC detector equipped with the L-curve stopping rule and based on the residual norm steepest descent and conjugate gradient least squares, respectively (which are referred to by RNSD-LPIC and CGLS-LPIC detectors, for conciseness).
First, we show how the L-curve method works. For this reason, we plot the norm of the residual error of both the RNSD-LPIC and CGLS-LPIC detectors for one OFDM symbol versus the stage index $p$, and we plot also its curvature using Equation (32). We set the carrier frequency to $3.5 \mathrm{GHz}, \mathrm{SNR}$ to $5 \mathrm{~dB}, N$ to 128 , and $K$ to 4 with frequency offsets $\left(\varepsilon_{1}=-0.35, \varepsilon_{2}=0.38, \varepsilon_{3}=0.36\right.$, $\left.\varepsilon_{4}=-0.39\right)$ and speeds $80,120,90$, and $100 \mathrm{~km} / \mathrm{h}$, respectively.

While a unique peak can be distinguished for the curvature of the residual's norm of the RNSD-LPIC detector as depicted in Figure 5, this is not the case with CGLS-LPIC detector. As shown in Figure 6, the residual's norm of the CGLS-LPIC detector exhibits an erratic behavior at later stages where it fluctuates and introduces wrong peaks in the curvature curve resulting in wrong stage index calculation using the L-curve method. To overcome this phenomenon, the norm of the residual error is fitted with a decaying exponential of the form: $a_{1}+a_{2} e^{-a_{3} p}$. It is clear now that the L-curve method can be used without any pitfall and a unique peak is obtained from the curvature information. In all subsequent simulations, we consider RNSD-LPIC and CGLS-LPIC detectors in which the norm of the residual error is fitted with the decaying exponential stated above.

In Figures 7, 8, and 9, the sensitivity of the L-curve method with respect to the condition number of the system matrix (which varies with the time varying fading channel) is evaluated for different SNRs. The same parameters used in Figures 5 and 6 are used here as well except for the SNRs where the SNR is fixed to $1,10,20$, and $30 \mathrm{~dB}$, respectively. In addition, the L-curve technique is used over 5,000 consecutive OFDM symbols and the histogram of the stage indices of the RNSD-LPIC and
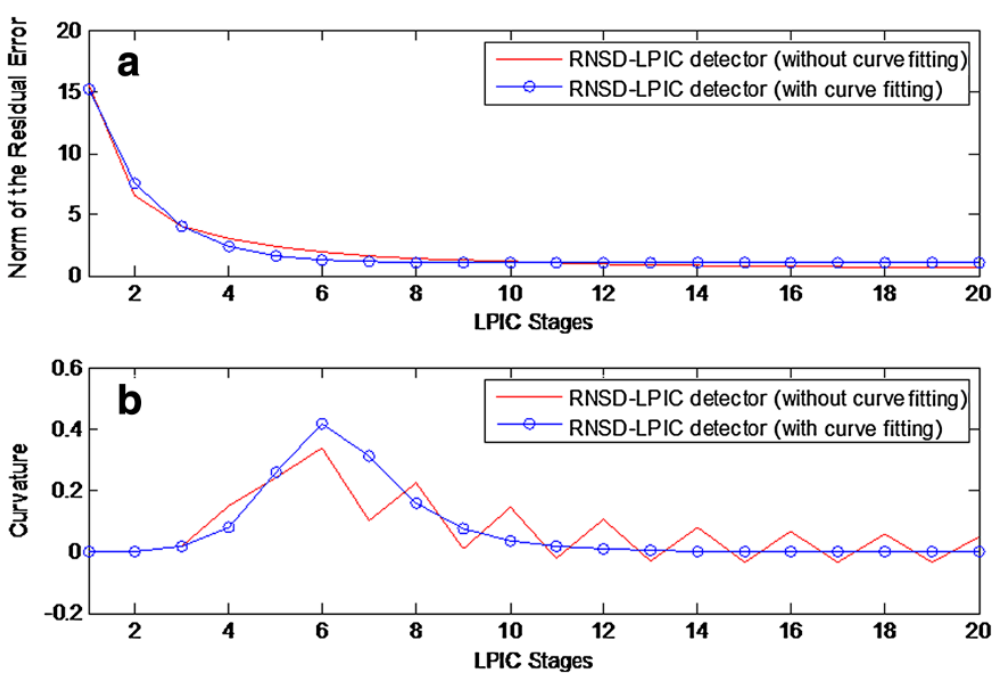

Figure 5 The (a) residual's norm and (b) its curvature of the RNSD-LPIC detector. 

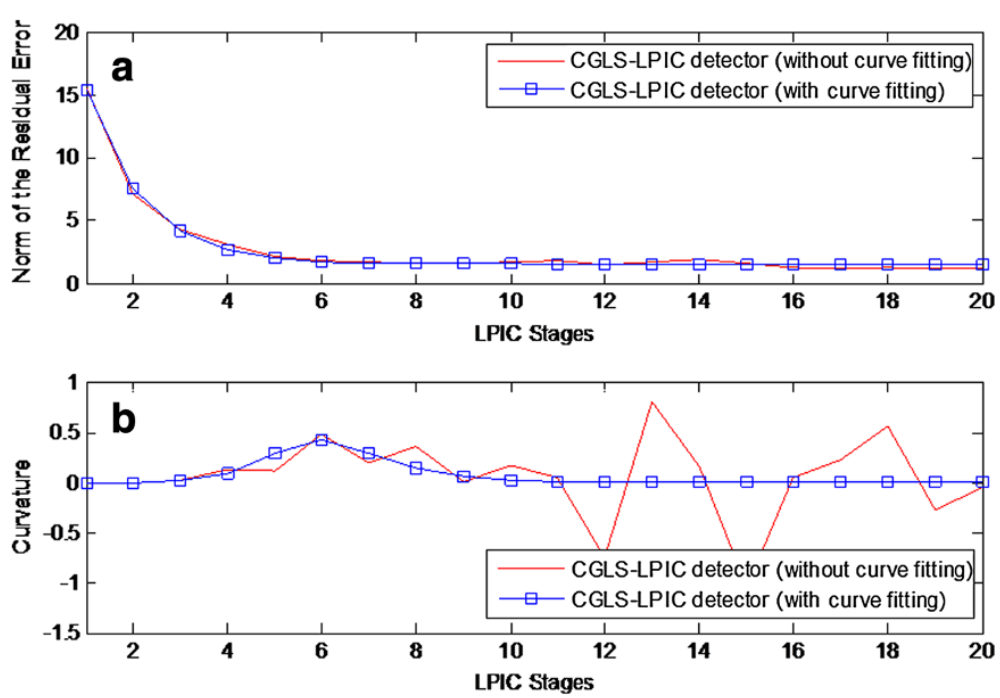

Figure 6 The (a) residual's norm and (b) its curvature of the CGLS-LPIC detector.

CGLS-LPIC detectors are plotted in Figures 8 and 9, respectively.

As depicted in Figure 7, the condition number of the system matrix varies from one OFDM symbol to another and it is dependent on the fading channels of the different users. It can be seen that the condition number of the system matrix varies largely in magnitude where it can go from $10^{3}$ to $10^{6}$.

As depicted in Figures 8 and 9, it is clear that the stopping stage determined by the L-curve method for both the RNSD-LPIC and CGLS-LPIC detectors is almost fixed with respect to the condition number variations of the system matrix over 5,000 OFDM symbol (see Figure 7) for each SNR. Consequently, it is sufficient to evaluate the stopping stage index for a few OFDM symbols (say 100 OFDM symbol) and then get the average stopping stage index and use it for the subsequent OFDM symbols. This is a very important result as it allows avoiding the calculation of the stopping stage for every OFDM symbol and thus renders this scheme very efficient in terms of computational complexity.

It also is clear that the stopping stage is almost constant on average with increasing SNR while in principle, it should increase. This is one of the deficiencies of the Lcurve method, and it is due to the fact that the L-curve method is not a converging stopping rule, in the sense that

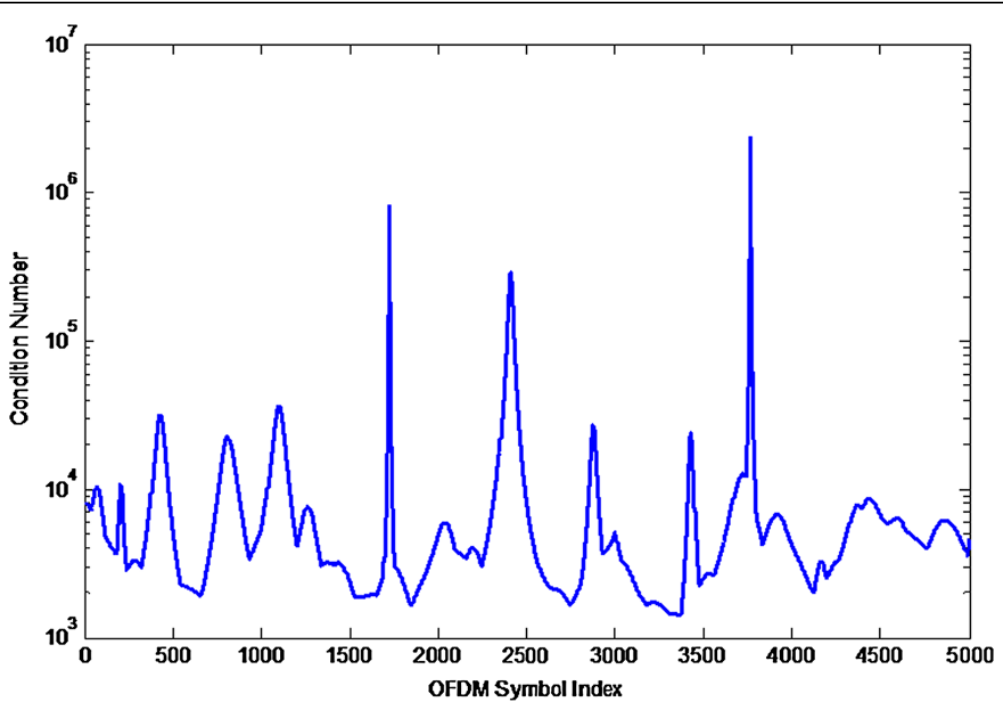

Figure 7 Channel condition number versus the OFDM symbol. 

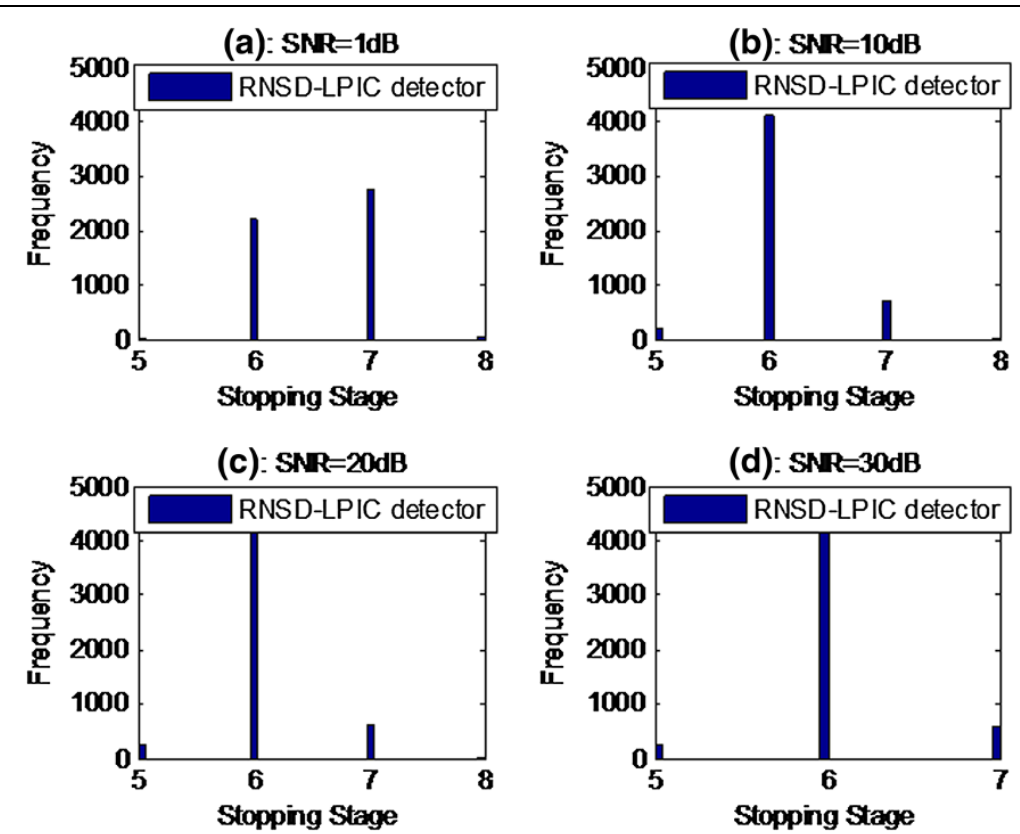

Figure 8 Histogram of the stopping stage index for $S N R=$ (a) $1 \mathrm{~dB}$, (b) $10 \mathrm{~dB}$, (c) $20 \mathrm{~dB}$, and (d) $30 \mathrm{~dB}$.

the solution obtained using the L-curve method does not converge to the true solution if the noise variance vanishes to zero.

The L-curve method is somewhat sensitive to the size of the system matrix determined by the number of subcarriers $N$. To illustrate this fact, we plot the histogram of the stage indices determined by the L-curve method over 5,000 OFDM symbols for two values of the subcarriers $N$, that is $N=8$ and $N=32$, respectively. We can see from Figures 10 and 11 that the most dominant stage index for $N=8$ is four stages while the most dominant stage index for $N=32$ is five stages. In addition, we have seen previously from Figures 8 and 9 that for $N=128$, the most dominant stage index is six stages which indicates that the most dominant stage index increases with the number of subcarriers $N$.
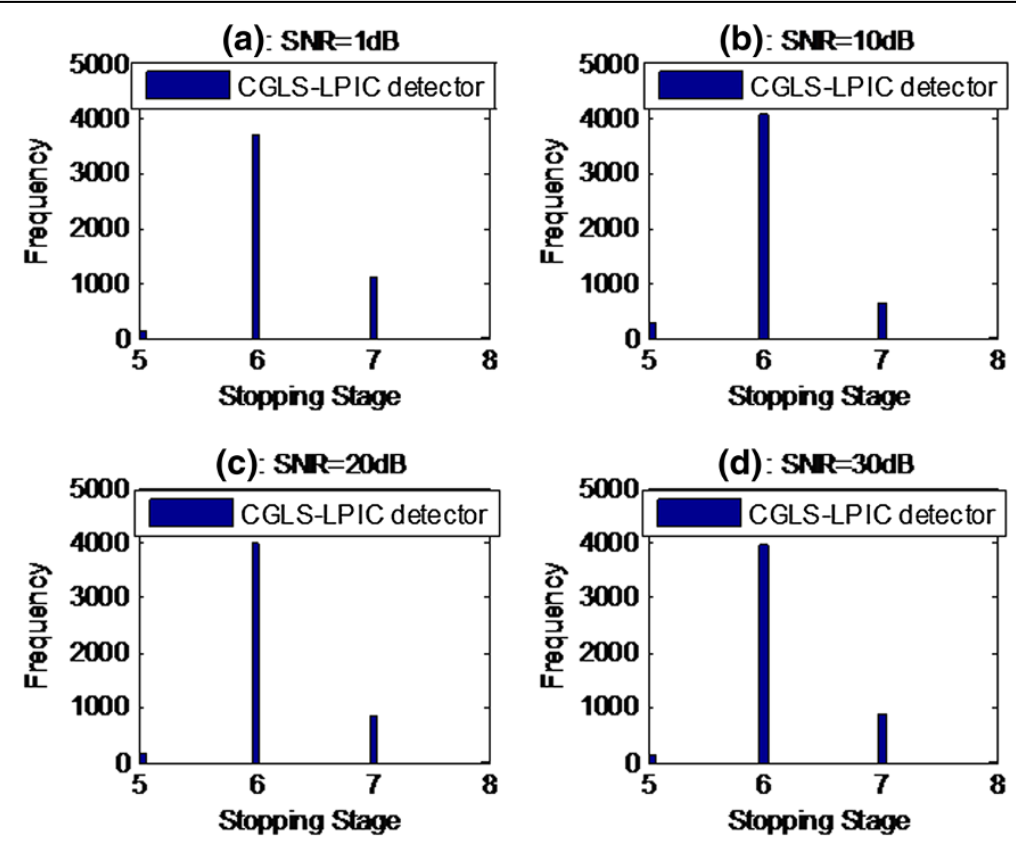

Figure 9 Histogram of the stopping stage index for $S N R=$ (a) $1 \mathrm{~dB}$, (b) $10 \mathrm{~dB}$, (c) $20 \mathrm{~dB}$, and (d) $30 \mathrm{~dB}$. 


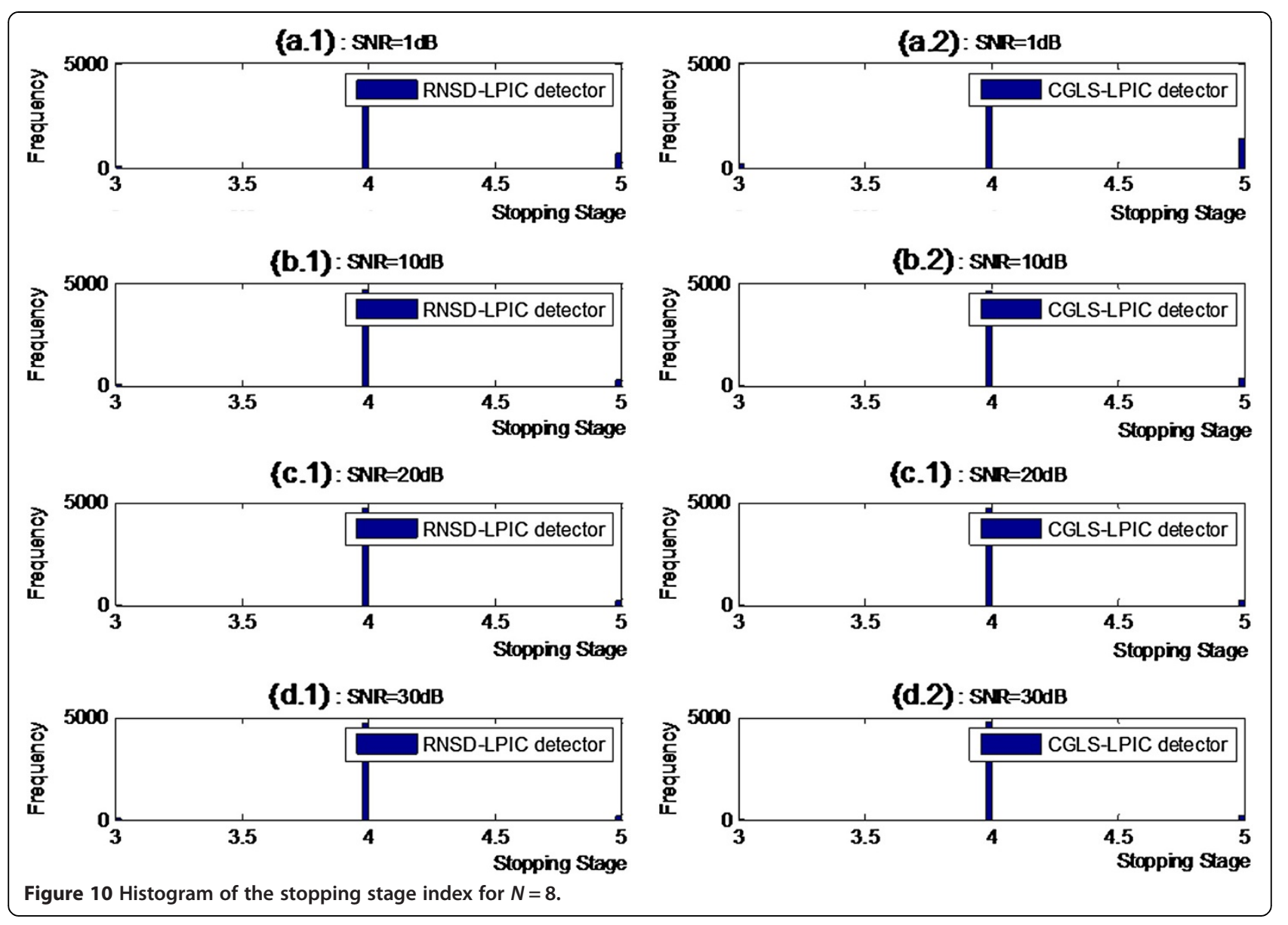

This suggests that the stopping stage determined using the L-curve method should be updated whenever the total number of subcarriers $N$ in the system changes, for example, if one user needs a higher data rate and is assigned a higher number of subcarriers, or if one user enters or leaves the system, etc.

In Figure 12, we evaluate the convergence behavior of both the RNSD-LPIC and the CGLS-LPIC detectors equipped by the L-curve stopping rule by varying the stage index $p$ and assessing its average BER performance. For comparison purposes, we simulate also the decorrelator (DEC) and the LMMSE (LMMSE) detectors. We use the same simulation parameters as in Figures 5 and 6 but we set the SNR to $13 \mathrm{~dB}$. One can see the semiconvergence behavior of both the RNSD-LPIC and the CGLS-LPIC detectors and how the detectors equipped with the L-curve method stop at six or seven stages and yield an average BER that is between that of the decorrelator and LMMSE detectors yet they do not require any information about the noise variance.

Figure 13 illustrates the average BER performance versus the SNR of the RNSD-LPIC and the CGLS-LPIC detectors equipped by the L-curve stopping rule. It is clear that the RNSD-LPIC detector performs better than the decorrelator detector if the SNR is less than $15 \mathrm{~dB}$ and the CGLS-LPIC detector performs better than the decorrelator detector if the SNR is less than $16 \mathrm{~dB}$. This suggests that the L-curve method can be used only for low to medium SNRs; fortunately, these regions suffer the most from the noise enhancement effect, and therefore, this deficiency of the L-curve method is not serious.

\section{Conclusion}

In this work, we introduced new linear interference cancellation detectors for low to medium SNR illconditioned communication systems that perform close to the LMMSE detector though they do not require the knowledge of the noise variance information. These linear IC detectors are based on early an stopping rule known as the L-curve method. Simulation results indicate that these detectors are insensitive to both the condition number of the system matrix and SNR and can work well up to SNR of $16 \mathrm{~dB}$.

\section{Appendix}

In the following, we develop the average BER of the RNSD-LPIC and CGLS-LPIC detectors. 


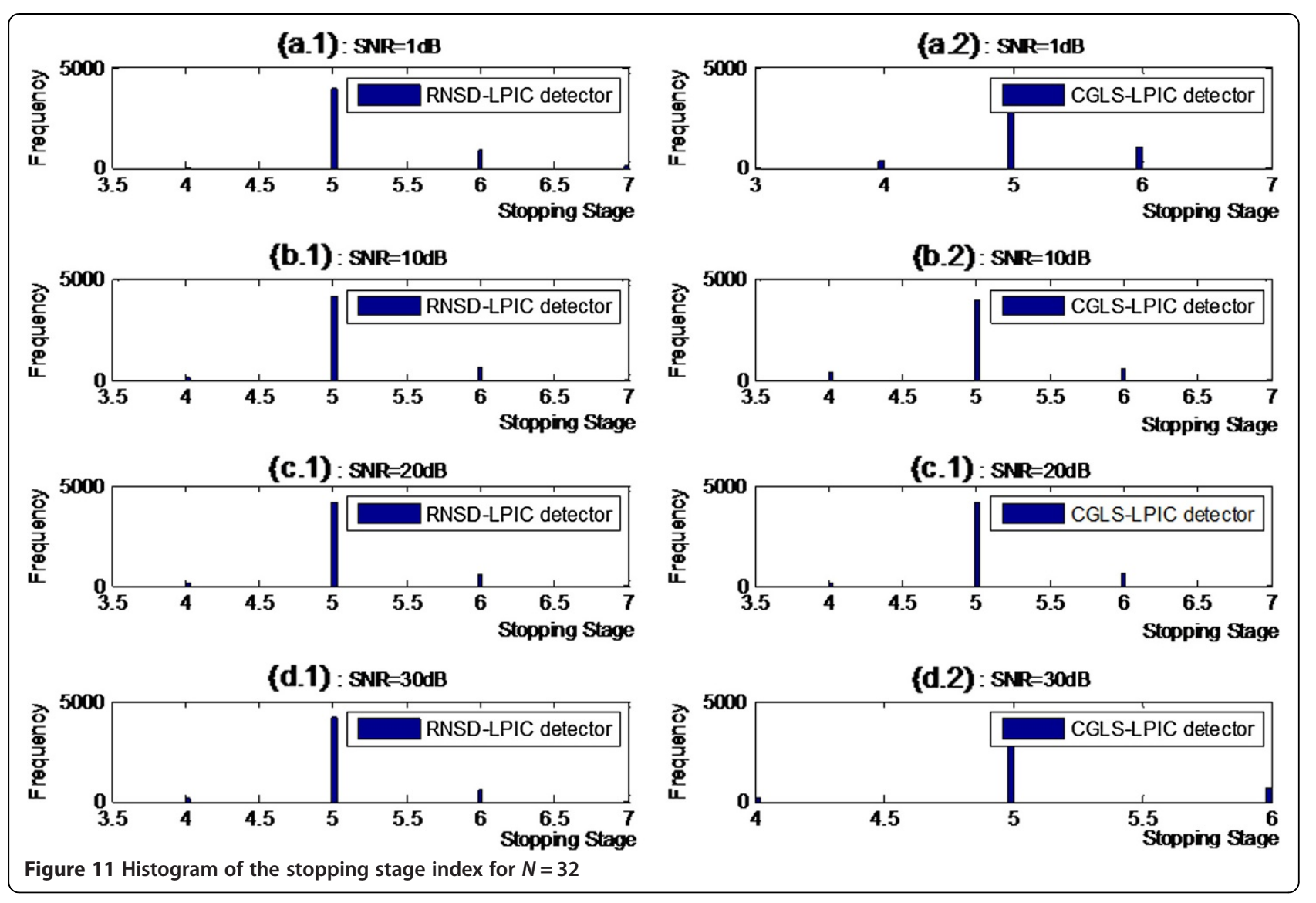

The QPSK scheme can be seen as two superimposed BPSK channels, these channels are orthogonal to each other and do not mutually interfere; therefore, the BER of QPSK scheme is simply the same as that of the BPSK scheme, and it is enough to conduct the BER probability analysis using the real or imaginary part only. In this derivation, we consider the real part of the decision vector $\mathbf{y}_{p}$.

The vectors $\mathbf{y}_{p}$ and $\mathbf{b}$ can be decomposed as $\mathbf{y}_{p}=$

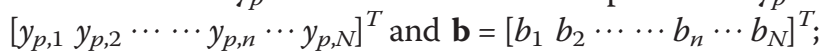

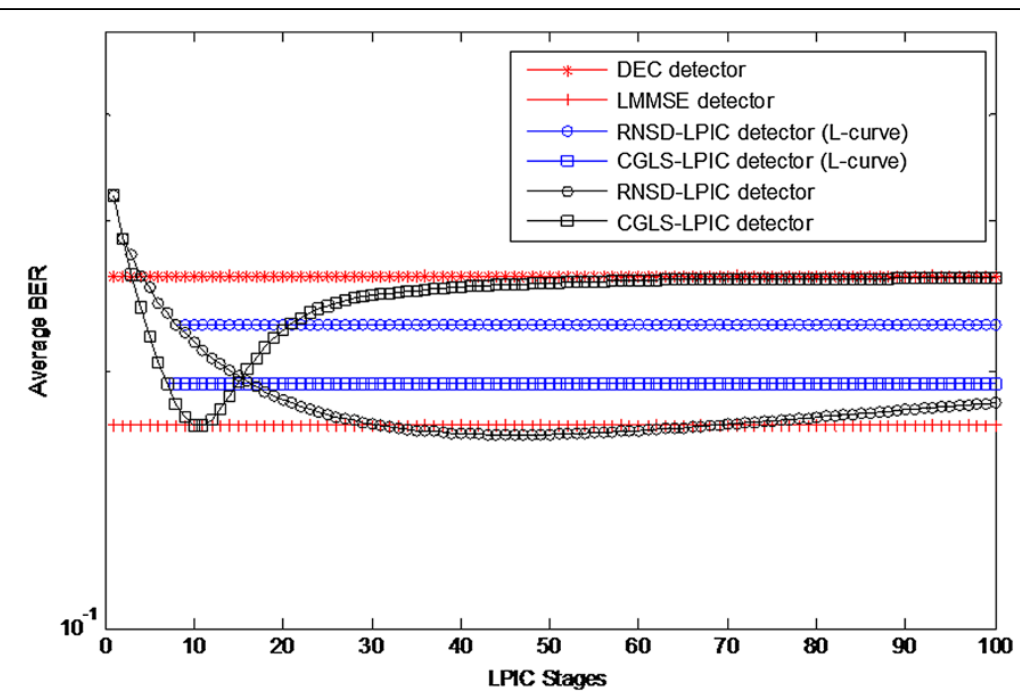

Figure 12 Convergence behavior of the RNSD-LPIC and the CGLS-LPIC detectors equipped by the L-curve stopping rule. 


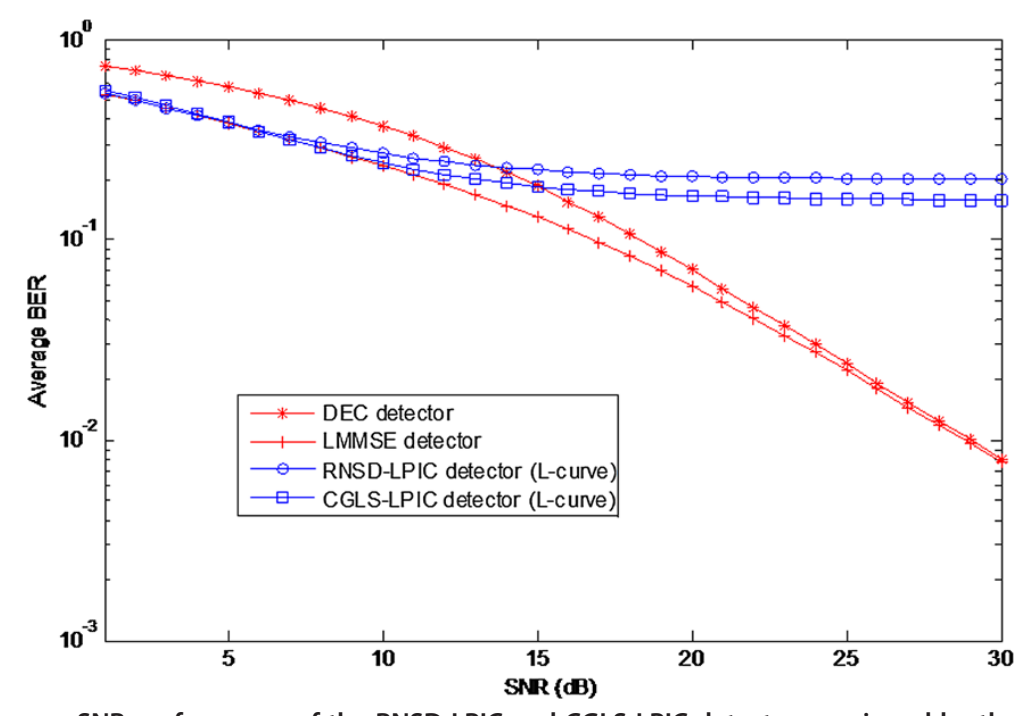

Figure 13 Average BER versus SNR performance of the RNSD-LPIC and CGLS-LPIC detectors equipped by the L-curve stopping rule.

therefore, the real part of the $n$th element of the vector $\mathbf{y}_{p}$ can be written as:

$$
\mathfrak{R}\left(y_{p, n}\right)=\mathfrak{R}\left(\mathbf{g}_{p, n}^{H} \mathbf{r}\right)=\mathfrak{R}\left(\mathbf{g}_{p, n}^{H}(\tilde{\tilde{\Psi}} \mathbf{b}+\mathbf{n})\right)
$$

where $\mathbf{n}$ is a vector of AWGN I.I.D samples of zero mean and variance $\rho^{2}$. By using the total probability theorem, we can write.

$$
\begin{aligned}
P\left(b_{n} \neq \widehat{b}_{n}\right)= & P\left(b_{n}=1, \mathfrak{R}\left(y_{p, n}\right)<0\right) \\
& +P\left(b_{n}=-1, \mathfrak{R}\left(y_{p, n}\right)>0\right) \\
= & P\left(b_{n}=1\right) P\left(\mathfrak{R}\left(y_{p, n}\right)<0 \mid b_{n}=1\right) \\
& +P\left(b_{n}=-1\right) P\left(\mathfrak{R}\left(y_{p, n}\right)>0 \mid b_{n}=-1\right) \\
= & \frac{1}{2} P\left(\mathfrak{R}\left(y_{p, n}\right)<0 \mid b_{n}=1\right) \\
& +\frac{1}{2} P\left(\Re\left(y_{p, n}\right)>0 \mid b_{n}=-1\right) \\
= & P\left(\mathfrak{R}\left(y_{p, n}\right)<0 \mid b_{n}=1\right)
\end{aligned}
$$

thus

$$
\begin{aligned}
P\left(b_{n} \neq \widehat{b}_{n}\right) & =P\left(\mathfrak{R}\left(\mathbf{g}_{p, n}^{H}(\tilde{\tilde{\mathbf{\Psi}}} \mathbf{b}+\mathbf{n})\right)<0 \mid b_{n}=1\right) \\
& =P\left(\mathfrak{R}\left(\mathbf{g}_{p, n}^{H} \mathbf{n}\right)<-\mathfrak{R}\left(\mathbf{g}_{p, n}^{H} \tilde{\tilde{\mathbf{\Psi}} \mathbf{b}}\right) \mid b_{n}=1\right)
\end{aligned}
$$

Due to the symmetry of the Gaussian (Normal) function, we have

$$
\begin{aligned}
P\left(\mathfrak{R}\left(\mathbf{g}_{p, n}^{H} \mathbf{n}\right)\right. & \left.<-\mathfrak{R}\left(\mathbf{g}_{p, n}^{H} \tilde{\tilde{\mathbf{\Psi}} \mathbf{b}}\right) \mid b_{n}=1\right) \\
& =P\left(\mathfrak{R}\left(\mathbf{g}_{p, n}^{H} \mathbf{n}\right)>\mathfrak{R}\left(\mathbf{g}_{p, n}^{H} \tilde{\tilde{\Psi}} \mathbf{b}\right) \mid b_{n}=1\right)
\end{aligned}
$$

hence

$$
\begin{aligned}
P\left(b_{n} \neq \widehat{b}_{n}\right) & =P\left(\mathfrak{R}\left(\mathbf{g}_{p, n}^{H} \mathbf{n}\right)>\mathfrak{R}\left(\mathbf{g}_{p, n}^{H} \tilde{\tilde{\Psi}} \mathbf{b}\right) \mid b_{n}=1\right) \\
& =Q\left(\frac{\mathfrak{R}\left(\mathbf{g}_{p, n}^{H} \tilde{\tilde{\Psi}} \mathbf{b}\right)-E\left[\Re\left(\mathbf{g}_{p, n}^{H} \mathbf{n}\right)\right]}{\sqrt{\operatorname{var}\left[\mathfrak{R}\left(\mathbf{g}_{p, n}^{H} \mathbf{n}\right)\right]}}\right) .
\end{aligned}
$$

We have

$$
E\left[\Re\left(\mathbf{g}_{p, n}^{H} \mathbf{n}\right)\right]=0
$$

and

$$
\begin{aligned}
\operatorname{var}\left[\Re\left(\mathbf{g}_{p, n}^{H} \mathbf{n}\right)\right] & =E\left[\mathfrak{R}\left(\mathbf{g}_{p, n}^{H} \mathbf{n}\left(\mathbf{g}_{p, n}^{H} \mathbf{n}\right)^{H}\right)\right] \\
= & E\left[\Re\left(\mathbf{g}_{p, n}^{H} \mathbf{n n}^{H} \mathbf{g}_{p, n}\right)\right] \\
= & \sigma^{2} \mathfrak{R}\left(\mathbf{g}_{p, n}^{H} \mathbf{g}_{p, n}\right),
\end{aligned}
$$


thus

$$
\begin{aligned}
P\left(b_{n} \neq \widehat{b}_{n}\right) & =Q\left(\frac{\mathfrak{R}\left(\mathbf{g}_{p, n}^{H} \tilde{\tilde{\Psi}} \mathbf{b}\right)-E\left[\Re\left(\mathbf{g}_{p, n}^{H} \mathbf{n}\right)\right]}{\sqrt{\operatorname{var}\left[\mathfrak{R}\left(\mathbf{g}_{p, n}^{H} \mathbf{n}\right)\right]}}\right) \\
& =Q\left(\frac{\Re\left(\mathbf{g}_{p, n}^{H} \tilde{\tilde{\Psi}} \mathbf{b}\right)}{\rho \sqrt{\mathfrak{R}\left(\mathbf{g}_{p, n}^{H} \mathbf{g}_{p, n}\right)}}\right) .
\end{aligned}
$$

Conditioning over all interfering bits, the probability of error can be written as:

$$
\begin{aligned}
P_{b}(p, n) & =\sum_{b_{1} \in\{-1,1\}} \cdots \sum_{\substack{b_{j} \in\{-1,1\} \\
j \neq n}} \ldots \sum_{b_{N} \in\{-1,1\}} Q\left(\frac{\mathfrak{R}\left(\mathbf{g}_{p, n}^{H} \tilde{\tilde{\Psi}} \mathbf{b}\right)}{\rho \sqrt{\Re\left(\mathbf{g}_{p, n}^{H} \mathbf{g}_{p, n}\right)}}\right) \\
& =\frac{1}{2^{N-1}} \sum_{\substack{\text { all } \\
b_{n}=1}} Q\left(\frac{\Re\left(\mathbf{g}_{p, n}^{H} \tilde{\tilde{\Psi}} \mathbf{b}\right)}{\rho \sqrt{\mathfrak{R}\left(\mathbf{g}_{p, n}^{H} \mathbf{g}_{p, n}\right)}}\right) .
\end{aligned}
$$

Averaging over all subcarriers, we obtain

$$
P_{b}(p)=\frac{1}{N} \sum_{n=1}^{N} \frac{1}{2^{N-1}} \sum_{\substack{\text { all } \mathbf{b} \\ b_{n}=1}} Q\left(\frac{\Re\left(\mathbf{g}_{p, n}^{H} \tilde{\tilde{\Psi}} \mathbf{b}\right)}{\rho \sqrt{\mathfrak{R}\left(\mathbf{g}_{p, n}^{H} \mathbf{g}_{p, n}\right)}}\right) .
$$

\section{Endnote}

${ }^{a}$ cflop states for any complex addition, complex subtraction, complex multiplication, or complex division.

\section{Competing interests}

The authors declare that they have no competing interests.

\section{Author details}

'Prince Sultan Advanced Technologies Research Institute (PSATRI), King Saud University, P.O. Box 800, Riyadh 11421, Saudi Arabia. ${ }^{2}$ KACST-Technology Innovation Center in Radio Frequency and Photonics for e-Society (RFTONICS), Electrical Engineering Department, King Saud University, P.O. Box 800, Riyadh 11421, Saudi Arabia.

Received: 9 March 2013 Accepted: 19 November 2013

Published: 5 December 2013

\section{References}

1. ML Honig, Advances in multiuser detection (Wiley Series in

Telecommunications and Signal Processing, Hoboken, NJ, 2009)

2. Z Peng, Z Xu, W Furong, $X X \mathbf{X}, T$ Lai, A relay assignment algorithm with interference mitigation for cooperative communication, in WCNC. Budapest 5-8, 1286-1291 (2009)

3. SW Hou, CC Ko, Intercarrier interference suppression for OFDMA uplink in time- and frequency-selective fading channels. IEEE. Tran. Vehicular. Technol. 58(6), 2741-2754 (2009)

4. M Takanashi, T Nishimura, Y Ogawa, T Ohgane, MIMO-UWB systems with parallel interference canceller using timing control scheme in LOS environments. in IEEE. Wireless. Commun. Netw. Conf. (2007), Kowloon, 11-15 March 2007, pp: 1599-1603

5. H Mrabet, I Dayoub, R Attia, S Haxha, Performance improving of OCDMA system using 2-D optical codes with optical SIC receiver. J. Lightwave. Technol. 27(21), 4744-4753 (2009)

6. E Axell, EG Larsson, Optimal and sub-optimal spectrum sensing of OFDM signals in known and unknown noise variance. IEEE. J. Select. Areas. Commun. 29(2), 290-304 (2011)

7. EG Larsson, R Thobaben, G Wang, On diversity combining with unknown channel state information and unknown noise variance. in Proc. of IEEE Wireless Communications and Networking Conference (WCNC), Sydney, 18-21 April 2010

8. B Abdelouahab, SA Alshebeili, Regularization property of linear interference cancellation detectors. EURASIP. J. Adv. Signal. Proc. 2012, 145 (2012)

9. U Hamarik, R Palm, On rules for stopping the conjugate gradient type methods in ill-posed problems. Math. Model. Anal. 12(1), 61-70 (2007)

10. IEEE Standard for Local and Metropolitan area networks Part 16, Air interface for fixed and mobile broadband wireless access systems amendment 2: physical and medium access control layers for combined fixed and mobile operation in licensed bands, 802nd edn (The Institute of Electrical and Electronics Engineering, Inc. Std. IEEE, Piscataway, NJ, 2006)

11. SK Hashemizadeh, H Saeedi-Sourck, MJ Omid, Sensitivity analysis of interleaved OFDMA system uplink to carrier frequency offset, in 2011 IEEE 22nd International Symposium on Personal Indoor and Mobile Radio Communications (, Toronto, ON, 2011), pp. 1631-1635

12. NM Thamban, Linear operator equations: approximation and regularization (World. Sci. Publ. Co., Singapore, 2009)

13. C Hansen, Rank-deficient and discrete ill-posed problems: numerical aspects of linear inversion (Society for Industrial and Applied Mathematics, Philadelphia, PA, 1998), pp. 403-6

14. J Nagy, K Palmer, Steepest descent, CG and iterative regularization of ill-posed problems. BIT 43, 1003-1017 (2003)

15. $P C$ Hansen, The $L$-curve and its use in the numerical treatment of inverse problems. Comput. Inverse. Prob. Electrocard. 2, 1-24 (2000)

16. PC Hansen, TK Jensen, G Rodriguez, An adaptive pruning algorithm for the discrete L-curve criterion. J. Comput. Appl. Math. 198(2), 483-492 (2006)

17. $\mathrm{P} \mathrm{Qu}, \mathrm{K}$ Zhong, B Zhang, J Wang, GX Shen, Convergence behavior of iterative SENSE reconstruction with non-Cartesian trajectories. Magn. Reson. Med. 54(4), 1040-5 (2005)

18. DR Joshi, DC Popescu, OA Dobre, Adaptive spectrum sensing with noise variance estimation for dynamic cognitive radio systems, in CISS 2010 (, Princeton, NJ, 2010), pp. 1-5

19. GH Golub, CF Van Loan, Matrix computations, 4th edn. (The Johns Hopkins University Press, Baltimore, MD, 2013)

doi:10.1186/1687-6180-2013-180

Cite this article as: Bentrcia and Alshebeili: Linear IC detectors for low to medium SNR ill-conditioned communication systems with unknown noise variance. EURASIP Journal on Advances in Signal Processing 2013 2013:180

\section{Submit your manuscript to a SpringerOpen ${ }^{\odot}$ journal and benefit from:}

- Convenient online submission

- Rigorous peer review

- Immediate publication on acceptance

- Open access: articles freely available online

- High visibility within the field

- Retaining the copyright to your article

Submit your next manuscript at $\gg$ springeropen.com 